\title{
Seawater attenuates blood lactate response to YoYo intermittent running test level 2 in euhydrated field-sport athletes - a crossover double-blind placebo trial
}

\author{
A. Walshe ${ }^{1}$, N. Ryan ${ }^{1}$, L. Ryan ${ }^{1}$ and E. Hannon ${ }^{1}$ \\ ${ }^{1}$ Department of Sport, Exercise, and Nutrition, School of Science and Computing, Galway-Mayo Institute of \\ Technology, Galway, Ireland
}

The current sports nutrition supplement market is being flooded with a plethora of products claiming to enhance athletic performance and wellbeing. Although stimulants and protein supplements are among the most common, electrolyte and carbohydrate-based hydration supplements are being marketed to Irish athletes and weekend warriors. This is exemplified by seawater (SW) supplements which have been proposed as an ergogenic hydration supplement in field-sports, both in Ireland and the $\mathrm{UK}^{(1)}$. Current SW research lacks external validity to said field-sports which therefore warrants further research.

The following study investigated the blood lactate (BLa) response to high-intensity intermittent running following SW ingestion via a YoYo Intermittent Recovery Test Level 2 (YoYo IR2). Nine amateur field-sport athletes completed a crossover, double-blind placebo study design, supplementing with SW or a taste-matched placebo (PL). BLa was assessed at baseline, and both intra and post-test. Assumption testing was performed and all data was analyzed using SPSS. A paired t-test assessed changes in BLa, and the BLa peak values obtained.

SW supplementation did not improve high-intensity intermittent running capacity $(\mathrm{p}>0.05)$. Significant differences existed between PL and SW at 2 minutes intra-test and 2 minutes post-test, $\mathrm{t}(8)=3.168, \mathrm{p}=0.013, \mathrm{~d}=0.79$, and at $4-6$ minutes post-test, $\mathrm{t}(8)=2.723$, $p=0.026, d=1.02$. BLaPeak was significantly lower in SW than PL, $t(8)=2.500, p=0.037, d=0.88$. These results indicate a significant effect of SW in attenuating the rate of BLa accumulation and BLaPeak following YoYo IR2 performance. Thus, the null hypothesis is rejected.

In this study, SW reduced the relative rate of lactate accumulation and BLaPeak. This improved lactate utilization may decrease the build-up of $[\mathrm{H}+]$ ions, delaying acidosis. Greater control of blood $\mathrm{pH}$ shifts the oxyhaemoglobin disassociation curve to the left, creating greater affinity through the Bohr effect and delaying muscular hypoxia. As fluid loss occurs naturally during sports performances, SW may also help offset the reduction of plasma volume experienced through its high $[\mathrm{Na}+]$ concentration. SW did attenuate the BLaPeak response to YoYo IR2 in the present study however this did not translate to increased performance outcomes. Further research with statistically significant samples is required to verify the efficacy of SW as an ergogenic aid in field-sport athletes.

\section{References}

1. Perez-Turpin JA, Trottini M, Chinchilla-Mira JJ, et al. (2017) Biol Sport 34(4), 407-412.

2. Higgins MF, Rudkin B \& Kuo CH (2019) Mar Drugs 17(5), 309.

3. Bishop D, Edge J, Thomas C, et al. (2008) Am J Physiol Regul Integr Comp Physiol 295, 1991-1998.

4. Volianitis S, Bredmose PP, Nielsen HB, et al. (2016) FASEB J 30, 685. 